\title{
End-User Software Customization by Direct Manipulation of Tabular Data
}

\author{
Geoffrey Litt \\ Massachusetts Institute of Technology \\ Cambridge, MA, USA \\ glitt@mit.edu \\ Tyler Millis \\ Massachusetts Institute of Technology \\ Cambridge, MA, USA \\ tmillis@alum.mit.edu
}

\author{
Daniel Jackson \\ Massachusetts Institute of Technology \\ Cambridge, MA, USA \\ dnj@mit.edu \\ Jessica Quaye \\ Massachusetts Institute of Technology \\ Cambridge, MA, USA \\ jquaye@alum.mit.edu
}

\begin{abstract}
Customizing software should be as easy as using it. Unfortunately, most customization methods require users to abruptly shift from using a graphical interface to writing scripts in a programming language.

We introduce data-driven customization, a new way for end users to extend software by direct manipulation without doing traditional programming. We augment existing user interfaces with a table view showing the structured data inside the application. When users edit the table, their changes are reflected in the original UI. This simple model accommodates a spreadsheet formula language and custom data-editing widgets, providing enough power to implement a variety of useful extensions.

We illustrate the approach with Wildcard, a browser extension that implements data-driven customization on the web using web scraping. Through concrete examples, we show that this paradigm can support useful extensions to many real websites, and we share reflections from our experiences using the tool.

Finally, we share our broader vision for data-driven customization: a future where end users have more access to the data inside their applications, and can more flexibly repurpose that data as part of everyday software usage.
\end{abstract}

CCS Concepts: • Human-centered computing $\rightarrow$ Webbased interaction; • Software and its engineering $\rightarrow$ Integrated and visual development environments.

Keywords: end user programming, software customization, browser extensions, web automation, spreadsheets

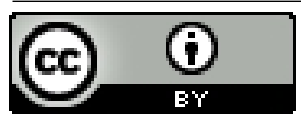

This work is licensed under a Creative Commons Attribution International 4.0 License.

Onward! '20, November 18-20, 2020, Virtual, USA

(C) 2020 Copyright held by the owner/author(s).

ACM ISBN 978-1-4503-8178-9/20/11.

https://doi.org/10.1145/3426428.3426914
ACM Reference Format:

Geoffrey Litt, Daniel Jackson, Tyler Millis, and Jessica Quaye. 2020. End-User Software Customization by Direct Manipulation of Tabular Data. In Proceedings of the 2020 ACM SIGPLAN International Symposium on New Ideas, New Paradigms, and Reflections on Programming and Software (Onward!'20), November 18-20, 2020, Virtual, USA. ACM, New York, NY, USA, 16 pages. https://doi.org/10.1145/ 3426428.3426914

\section{Introduction}

Many applications don't meet the precise needs of their users, and it is impossible for developers to anticipate everyone's unique requirements. End user customization systems can help close this gap, by empowering non-programmers to modify their software to satisfy their personal goals.

Many end user customization systems [7, 9, 10, 17] offer a scripting model. They use various strategies to make programming more approachable: friendly syntax, a visual programming environment, or macro recording to bootstrap from concrete demonstrations. But all these techniques build on the same fundamental foundation: an imperative programming model, with statements, mutable variables, and loops.

We have known for decades about an alternative: direct manipulation [21], where "visibility of the object of interest" replaces "complex command language syntax." Direct manipulation is the de facto standard in GUIs today, but when it comes to customizing those GUIs, it is rarely to be found. Switching from using an application to customizing it via scripting requires an abrupt shift in interaction model, and poses a steep learning barrier for users not familiar with programming.

We subscribe to MacLean et al.'s vision of a "gentle slope" [19] free of such "cliffs," where users should only need to make minimal and incremental investments in skill to achieve their desired customizations. We seek to contribute to this gentle slope with a new method for customizing software via direct manipulation, taking inspiration from visual database query interfaces and spreadsheets, which have successfully 


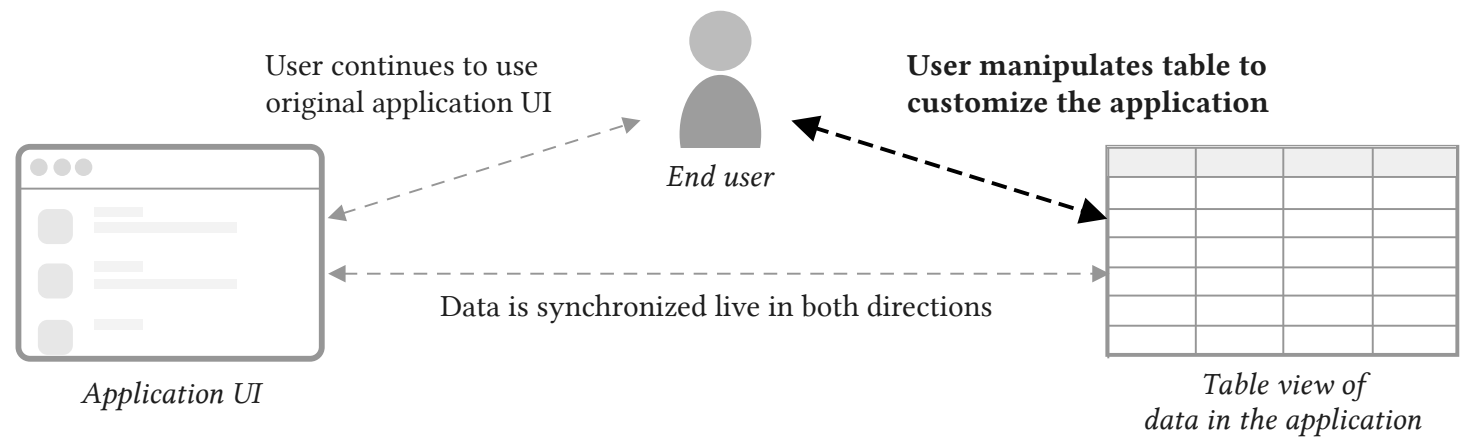

Figure 1. An overview of data-driven customization

enabled millions of end users to compute with data through direct manipulation.

In our proposed paradigm, data-driven customization, an application's UI is augmented with a table view where the user can see and manipulate the application's internal data. These changes don't just apply to the table; they also result in immediate changes to the application's original user interface. The user can sort/filter data in the UI, inject annotations, pull in related information from other web services, and more, all using the table as a mediating interface. Interacting with the table view resembles interacting with a familiar spreadsheet, but results in customizing an existing application.

To explore this idea in a real context, we have developed a browser extension called Wildcard that uses web scraping techniques to implement data-driven customization for existing Web applications. We introduce the tool with an example customization of Hacker News in Section 2, and then describe the implementation in Section 3. In Section 4, we present evidence that Wildcard can produce useful customizations, by sharing reflections from customizing 11 different websites in ways that met our own personal needs.

Wildcard is just an initial proof of concept of data-driven customization. In Section 5, we discuss our broader vision for how this style of customization could change the relationship between users and creators of software, focusing on three ideas:

- Decoupling data from applications: On the modern Web, data is often stored in proprietary silos, limiting the agency of users to choose their applications and flexibly work with data. We propose data-driven customization as an incremental step towards a more decentralized architecture, where users gain more control over the storage, processing and display of information from web services.

- Customization by direct manipulation: We explain how data-driven customization can provide a gentle slope, by allowing a user to customize an application by directly seeing and changing its data, rather than by writing imperative scripts.

- Semantic wrappers: Typically, tools that don't rely on official extension APIs resort to offering low-level APIs for customization. Instead, we propose a communitymaintained library of semantic wrappers around existing applications, enabling end users to work with domain data rather than low-level representations.

In Section 6 we discuss connections to related work. Our goals overlap with software customization tools, and our methods overlap with direct manipulation interfaces for working with structured data, including visual database query systems and spreadsheets. Finally, in Section 7, we conclude and describe opportunities for future work.

\section{Example Scenario}

To concretely illustrate the user experience of data-driven customization, we present a scenario of customizing Hacker News, a popular tech news aggregator. Figure 2 shows accompanying screenshots.

Opening the table: When the user opens Hacker News in a browser equipped with the Wildcard extension, they see a table at the bottom of the page. It contains a row for each link on the homepage, listing information like the title, URL, submitter username, number of points, and number of comments (Figure 2, Note A). The end user didn't need to do any work to create this table, because a programmer previously created an adapter to extract data from this particular website, and contributed it to a shared library of adapters integrated into Wildcard.

Sorting by points: First, the user decides to change the ranking of links on the homepage. Hacker News itself uses a ranking algorithm in which the position of an article depends not only on its point count (a measure of popularity), but also on how long it has been on the site. If the user hasn't been checking the site frequently, it's easy to miss a popular article that has fallen lower on the list. Sorting the page just by points would achieve a more stable ranking. 
A) Opening the table: The user opens a table view which shows data about each article in the list: its title, link, the number of points and comments, etc.

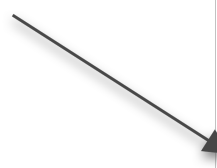

B) Sorting by points: When the user sorts the table by points, the web page becomes sorted in the same order

\section{C) Computing estimated} read times: The user enters a formula to fetch estimated read times from an API, and uses another formula to transform the results into a readable label

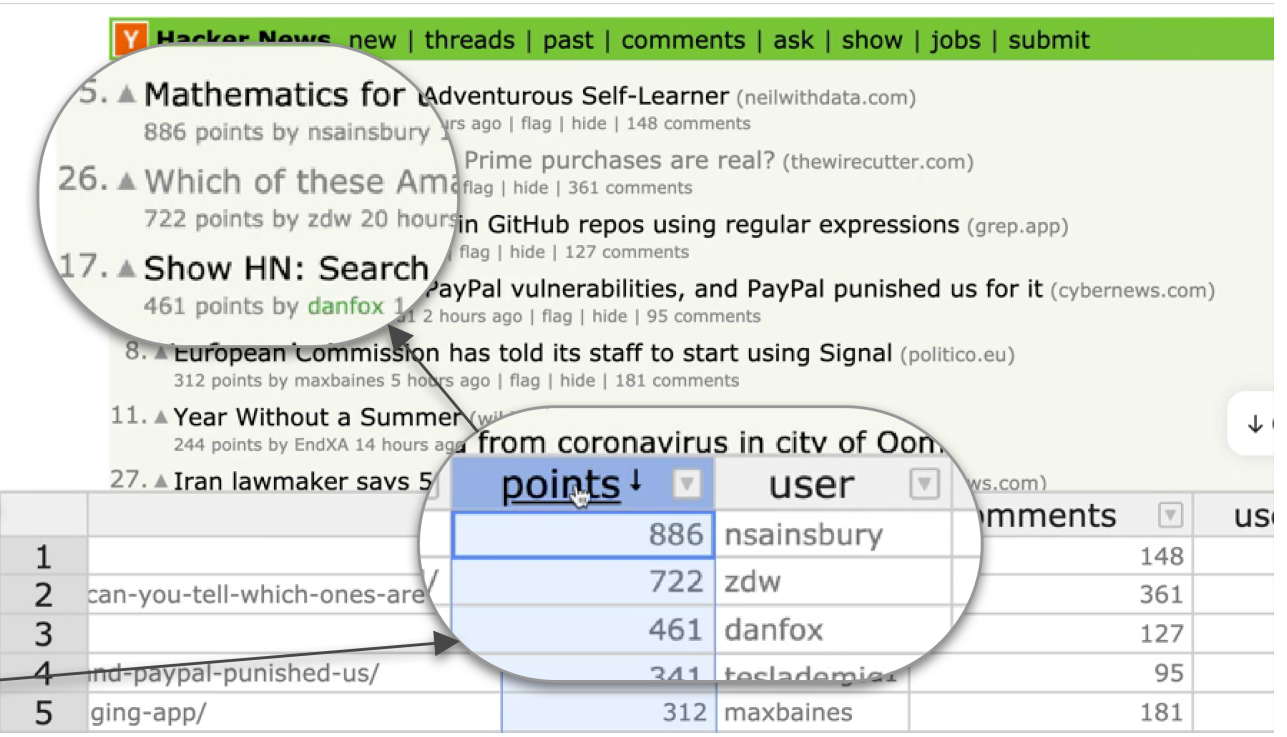

vs 50 dead from coronavirus in citv of Oom (aonews.com)

\begin{tabular}{|c|c|c|c|c|}
\hline$\nabla$ & user1 & user2 $\nabla$ & user3 & usel \\
\hline 148 & \multicolumn{2}{|c|}{$=$ ReadTimeInSeconds (link) } & & \\
\hline 361 & & & & \\
\hline 127 & & & & \\
\hline
\end{tabular}

ndxA 14 nours ago | rlag | nide | 98 comments | 14

ker savs 50 dead from coronavirus in citv of Oom (aonews.com)

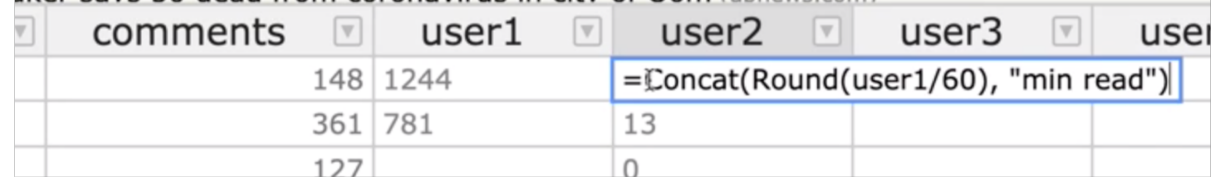

D) Showing read times: The formula results, and manual annotations, are shown in the page next to each article

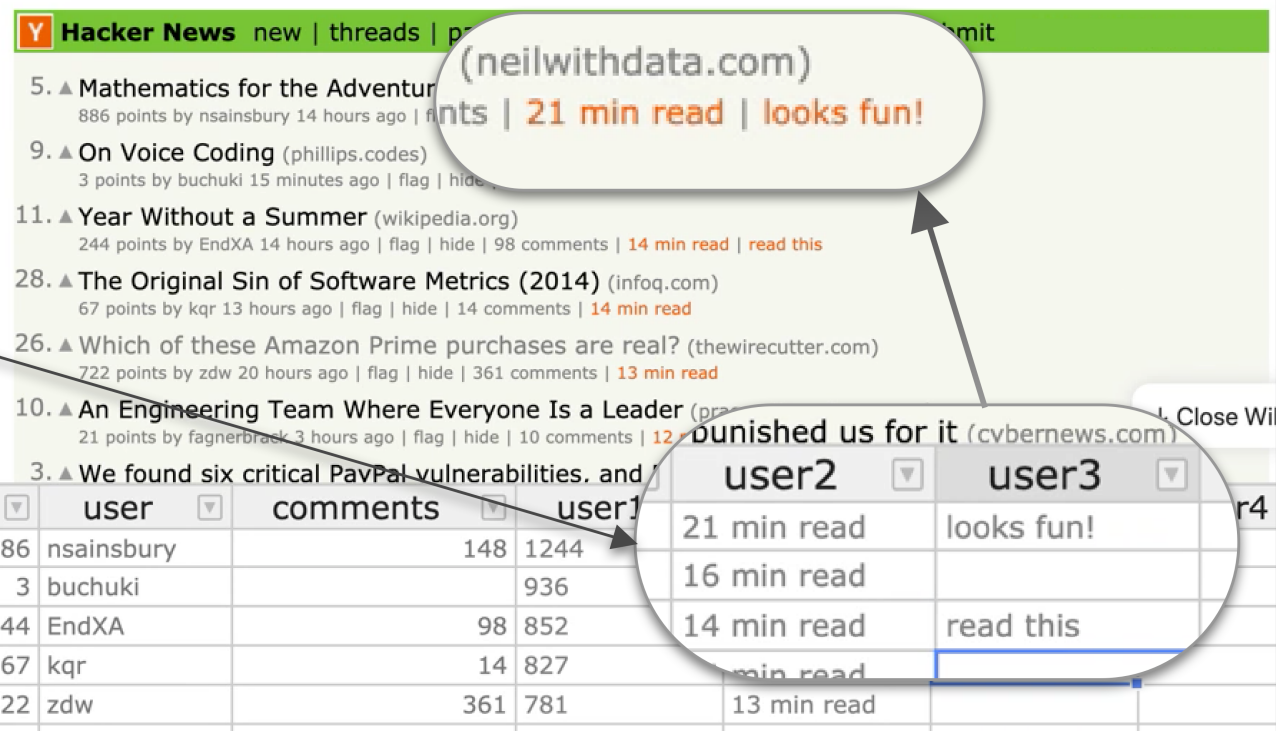

Figure 2. Customizing Hacker News by interacting with a table view 
To achieve this ordering, the user simply clicks on the "points" column header in the table. This sorts the table view by points, and the website UI also becomes sorted in the same order (Figure 2, Note B). Internally, Wildcard has changed the webpage's DOM to synchronize it with the sort order of the table. This sort predicate is also persisted in the browser and reapplied automatically the next time the user loads the page, so they can always browse the page sorted by points.

Adding estimated read times: Next, the user decides to attempt a more substantial customization: adding estimated read times to each article, in order to prioritize reading deeper content.

The table contains additional empty columns where the user can enter spreadsheet-like formulas to compute derived values. The user enters a formula into the first column for user-defined formulas or data, which is named user 1 by default (Figure 2, Note C): =ReadTimeInSeconds(link).

This formula calls a built-in function ReadTimeInSeconds that uses a third-party public web API to compute an estimated read time for the URL's contents. The link argument in the formula refers to a column name in the table; the formula is automatically evaluated across all rows in the table, using the value of link for each row.

The user clicks the user 1 column header to sort the articles on the page in descending order of estimated read time. They would also like to display the read times in the page, but a number in seconds isn't the most legible format, so they enter another formula in the user 2 column: $=$ Concat (Round (user $1 / 60$ ), "min read"). This formula converts seconds to minutes by dividing by 60 and rounding, then concatenates the result with a string label, producing results like " $21 \mathrm{~min}$ read".

Finally, the user clicks a menu option in the table header to display the contents of this new column in the original page (Figure 2, Note D). Each article on the page now shows an annotation with the estimated read time in minutes. (The formatting of annotations was determined by the programmer who created the adapter for Hacker News.)

Adding manual annotations: The user can manually add notes to the table, by entering data values into the table without formulas. In this case, the user jots down a few notes in another column about articles they might want to read, and the notes appear in the page next to the read times (Figure 2, Note D). The annotations are also stored in the browser's local storage so they can be retrieved on future visits.

Filtering out visited links: The user can filter out articles they have already read. (We omit this example from the figure for brevity.) The user can call a built-in function that returns a boolean depending on whether a URL is in the browser's history: =Visited(link).

They can then filter the table to only contain rows where this formula column contains false; links that the user has already visited are hidden both from the table view and the

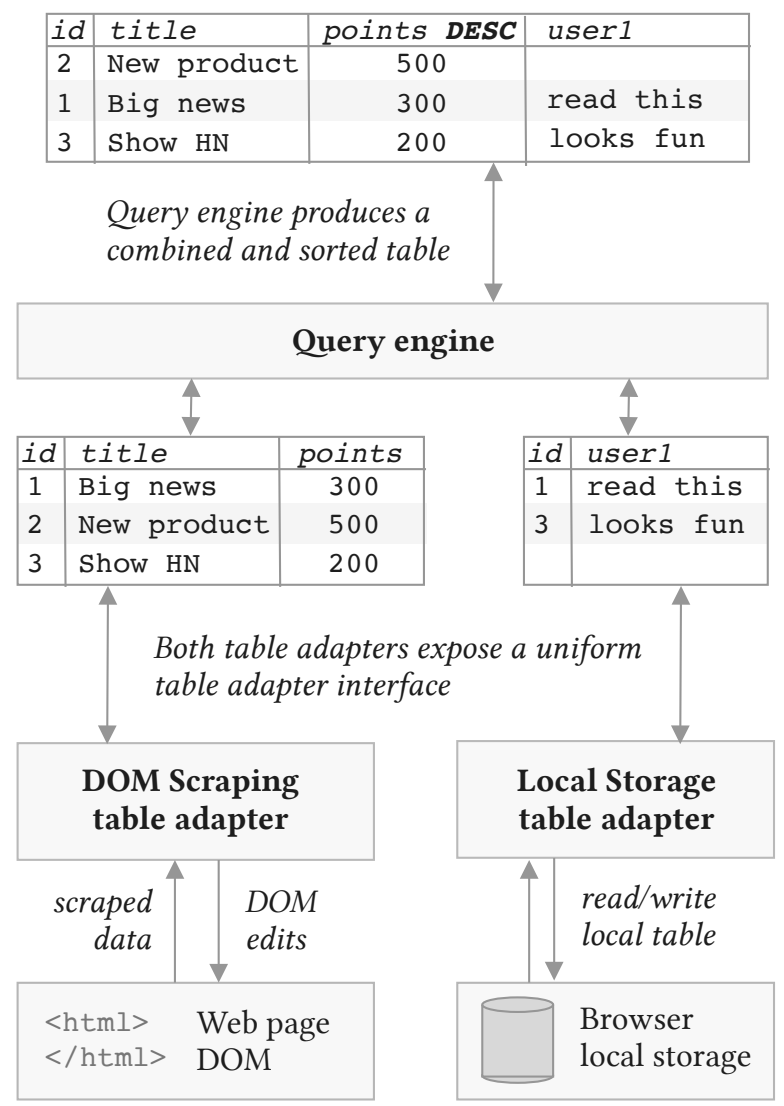

Figure 3. The table adapter architecture

original page. This is an example of a customization that the original website could not have implemented, since websites don't have access to the browser history for privacy reasons. But by using Wildcard, the user was able to implement the customization locally, without needing to expose their browser history to Hacker News.

This scenario has shown a few examples of how datadriven customizations empower a user to improve their experience of a website. Section 4 explains many other use cases and contexts where the technique applies, but first we explain how the system works internally.

\section{System Architecture}

Figure 3 summarizes the overall architecture of data-driven customization, using a simplified illustration of the Hacker News example scenario. The name and points value for each article is scraped from the web page DOM, and user annotations are loaded from the brower's local storage.

First, the web page and the browser storage are each wrapped by a table adapter, which defines a bidirectional mapping between an underlying data source and a table. In addition to a read mapping for how the underlying data 
should be represented as a table, it also has a write mapping defining the effects that edits have on the original data source.

The local storage adapter has a trivial mapping: it loads a table of data stored in the browser, and persists edits to that state. The mapping logic of the DOM scraping adapter is much more involved. It implements web scraping logic to produce a table of data from the web page, and turns edits into DOM manipulations, such as reordering rows of data on the page.

The two tables are then combined into a single table for the user to view and edit. The query engine is responsible for creating this combined view, and routing the user's edits back to the individual table adapters. In this example, the query engine has joined the two tables together by a shared ID column, and sorted the result by the points column.

We now examine each component of the system in more detail.

\subsection{Table Adapters}

A key idea in data-driven customization is that a wide variety of data sources can be mapped to a generic table abstraction. In a relational database, the table matches the underlying storage format, but in data-driven customization, the table is merely an interface layer. The data shown in the table is a projection of some underlying state, and edits to the table can have complex effects on the underlying state.

3.1.1 Abstract Interface. We begin by describing the abstract interface fulfilled by a table adapter.

Returning a table: A table adapter exposes a table of data: an ordered list of records. Each record carries a unique identifier and associates named attributes with values. Tables have a typed schema, so the same attributes are shared across all records. We currently support strings, numeric values, booleans, and datetimes as types. The columns also carry some additional metadata, such as whether or not they are read-only or editable.

A table adapter can update the contents of a table at any time in response to changes in the underlying state (e.g., a DOM scraping adapter can update the table when the page body changes). When data changes, the query view is reactively updated in response.

Handling edits: The query engine can issue a request to a table adapter to make an edit to a record. The meaning of making an edit can vary depending on the adapter: in the local storage adapter, a new value may be persisted into local storage; in the DOM scraping adapter, an edit may result in changing the value of a form field.

In addition, the query engine also sends additional information about the combined query view to each table adapter:

Sorting/filtering: When the user sorts or filters the query view, an ordered list of visible IDs is sent to each table adapter.
The DOM scraping adapter uses this information to change the list of rows shown in the web page.

Data from other tables: The query engine provides each table adapter with the entire combined table shown to the user. The DOM scraping adapter uses this for injecting annotationsvalues from other tables are added to the original web page.

Currently selected record: As the user clicks around the table view, the query engine broadcasts the record currently selected by the user to each table adapter. The DOM scraping adapter uses this information to highlight the row in the page that corresponds to the selected row in the table, which helps the user understand the mapping between the table and the original UI.

Next, we present the three types of table adapters we have built in Wildcard so far. These do not represent an exhaustive set of all possible table adapters-in Section 5 we discuss other types of adapters that would fit well into the general paradigm.

3.1.2 DOM Scraping Adapters. DOM scraping adapters enable Wildcard to interface with an existing website UI. A DOM scraping adapter fulfills the standard web scraping task of extracting a table of data from the DOM, but it also acts in the reverse direction: manipulating the DOM to reflect edits to the table.

In Wildcard, DOM scraping adapters are programmed manually for each website using Javascript code. It might seem that this prohibits non-programmer users from using the system at all, but we mitigate this problem with a shared repository of adapters. Once an adapter is programmed for a website, it is added to the shared repository, enabling any end user to perform customizations on that website.

In the future, other strategies for producing DOM scraping adapters could reduce this dependence on programmers: an end user could specify the scraping logic via demonstration, or the desired data table could be automatically inferred from the page. While we are interested in these techniques and discuss them in Section 5, we believe that a shared repository of manually programmed adapters is a pragmatic starting point; given that many users visit the same popular websites, a critical mass of adapters could serve the needs of many users.

To make it easier to create these adapters, Wildcard provides a framework that makes the process feel more like writing unidirectional scraping code than performing a complex bidirectional synchronization. The key idea is this: programmers return pointers to DOM elements representing table rows and table cells; Wildcard extracts data from these DOM elements, but it also uses the pointers to synchronize table edits back into the page. For example, when the user sorts the table, the DOM elements representing the table rows are moved around in the DOM to reflect the new sorted order. 


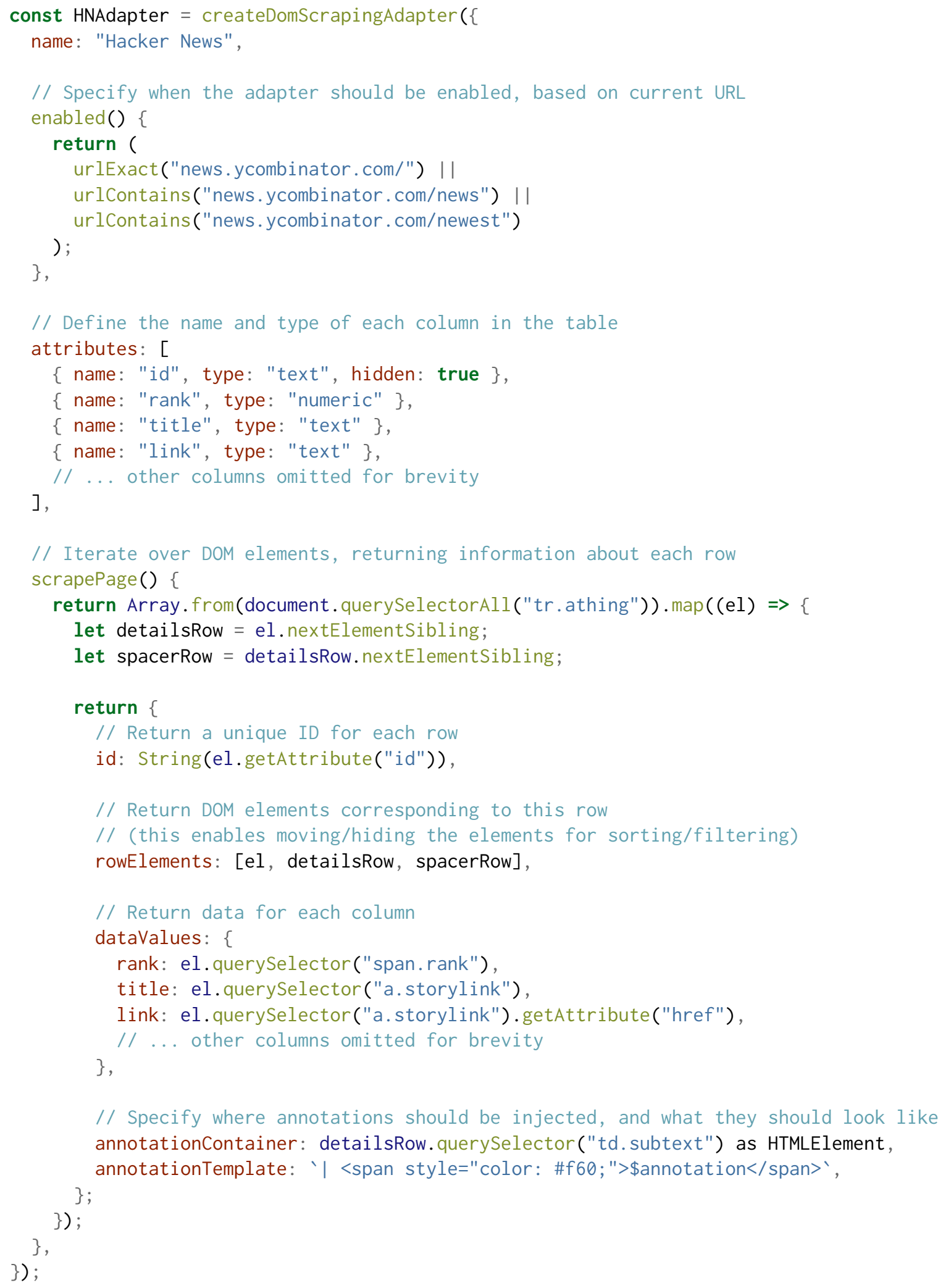

Figure 4. Source code for the Hacker News scraper. Some details removed for brevity. 
Figure 4 shows an example of the scraper code used for the Hacker News example (with some code eliminated for brevity.) It defines the following main components:

- enabled: defines when this adapter should run, usually based on the active URL in the browser. ${ }^{1}$

- attributes: defines a schema for the table, with a name and type for each column

- scrapePage: defines a scraping function which returns an array of objects, each containing the data for a single row of the table.

Here are some of the concerns that emerge when building adapters in practice:

Choosing a row ID: When possible, it is best to choose a server-side identifier that remains stable across pageloads. This enables user annotations persisted in local storage to be associated with the same records on subsequent pageloads. We have found that it's usually possible to find such an identifier; for example, each item in a page often contains a link to a page with more details, with a URL that contains a stable ID.

Types of scraped values: For each individual value within a row, there are two options for what type of data can be returned by the programmer-specified scraping function.

The default option is to return a DOM element, in which case the generic adapter extracts the text contents of the DOM element and casts them to the type of the column. The advantage of returning a DOM element is that the value is editable-when the user changes the value in the table, the generic adapter can simply overwrite the inner contents of the DOM element.

Another option is to directly return a value, rather than returning a DOM element. The advantage of this approach is that the adapter author can perform arbitrary computations to derive the returned value-for example, they can use a regular expression to extract a substring. The disadvantage is that the field is no longer writable. The computation used to derive the value isn't reversible, so there's no way to reflect a table edit in the DOM.

Optional overrides: In order to turn a unidirectional scraping function into a bidirectional scraping adapter, there are a number of behaviors that must be specified:

- when should the scraping function be re-run in response to changes on the page?

- how should injected annotations appear in table rows?

- when the user selects a row in the table, how should the corresponding row in the DOM be highlighted?

The scraping framework defines sensible defaults that work well on many sites, but the programmer can optionally override them to provide better site-specific behavior

\footnotetext{
${ }^{1}$ Currently Wildcard can only show a single table at a time, so if multiple adapters are enabled for a single page, we arbitrarily pick one. It would be a straightforward extension to allow the user to switch between multiple possible tables available on the page.
}

For example, the Hacker News adapter specifies annotation options that make user annotations appear more naturally in the design of the original webpage.

3.1.3 AJAX Scraping Adapters. An AJAX scraping adapter intercepts AJAX requests made by a web page, and extracts information from those requests to add to the table. When available, this tends to be a helpful technique because the data is already in a structured form so it is easier to scrape, and it often includes valuable information not shown in the UI.

As with DOM scraping adapters, we have made it easy for programmers to create site-specific AJAX scraping adapters. A programmer writes a function that specifies how to extract data from an AJAX request, and the framework handles the details of intercepting requests and calling the programmerdefined function. ${ }^{2}$

In order to join the tables produced by AJAX scraping and DOM scraping, a common set of identifiers is required across records in the two tables. Often there is a server-defined ID present both in the DOM and in AJAX responses; if not, the programmer can use some set of overlapping data (e.g. an item name) as a shared ID.

3.1.4 Local Storage Adapters. The local storage adapter simply stores a table of data in the browser. This is currently only used to persist annotations.

The table view is initialized with empty columns such as user 1 which serve as the user's "scratch space," as shown in Section 2. When the user makes edits to these columns, new rows are created in the local storage table. The rows contain the record ID from the DOM scraping adapter, which enables them to be re-associated with the same records on subsequent pageloads.

\subsection{Query Engine}

The query engine is responsible for coordinating across multiple table adapters. It joins data across multiple tables and creates a single result table which is shown to the user through the editor. It also handles all user interactions and routes appropriate messages to each table adapter.

Queries are processed in three steps. First, the query invokes a primary DOM scraping adapter that associates table rows with elements in the application's user interface. Next, additional tables (AJAX data, local storage data) are leftjoined by ID. Finally, the result table is sorted and filtered according to user-specified predicates.

One way to view this query model is as a tiny subset of the SQL query model. Despite its simplicity, this model has proven sufficient for meeting our customization needs, and minimizes the complexity of supporting arbitrary queries.

\footnotetext{
${ }^{2}$ So far we have only implemented AJAX scraping in the Firefox version of Wildcard, since Firefox has convenient APIs for intercepting requests. It appears possible to implement in Chrome and Edge as well, but we have not finished our implementation.
} 
But because it fits into the general paradigm of relational queries, it could theoretically be extended to support a wider range of queries.

The query engine is also responsible for executing formulas. We have built a small formula language resembling a spreadsheet formula language. As in visual database query tools like SIEUFERD [3] and Airtable, formulas automatically apply across an entire column of data, and reference other column names instead of values in specific rows. This is more convenient than needing to copy-paste a formula across an entire column as in spreadsheets, and has worked for all of the customizations we have built.

\subsection{Table Editor}

We provide a table editor view as the user interface on top of the query engine. Our table editor is built with the Handsontable Javascript library, which provides built-in UI elements for viewing, editing, sorting, and filtering a table.

In addition to the basic table editing operations, we also provide cell editors: UI widgets that expose a custom editing UI for a single cell of the table view. A programmer building a cell editor need only integrate it with the table viewer; propagating values into the website UI is handled by the site-specific DOM adapter. In Section 4 we provide some examples of using cell editors.

The table editor only serves as a shallow interface layer over the query engine, relaying user commands to the query engine and rendering the resulting data table. Because of this architectural split, it would be straightforward to develop additional table editor interfaces on top of the Wildcard system. For example, we could provide a calendar view for displaying a table containing a date column.

\section{Reflections on Usage}

To evaluate data-driven customization in practice, we built the Wildcard browser extension, which implements datadriven customization in the context of existing websites. It is implemented in Typescript, and works across three major browsers: Chrome, Firefox, and Edge.

We developed site-specific adapters for 11 websites that we personally use frequently, and then built customizations for those websites using the Wildcard table view. Table 1 summarizes these results, showing the number of lines of code in the adapter for each site, and some example customizations we created. Here we offer our reflections from these experiences using the system, focused on two key questions:

- How broad is the range of possible customizations in this paradigm?

- How feasible is it to build DOM scraping adapters for real websites?

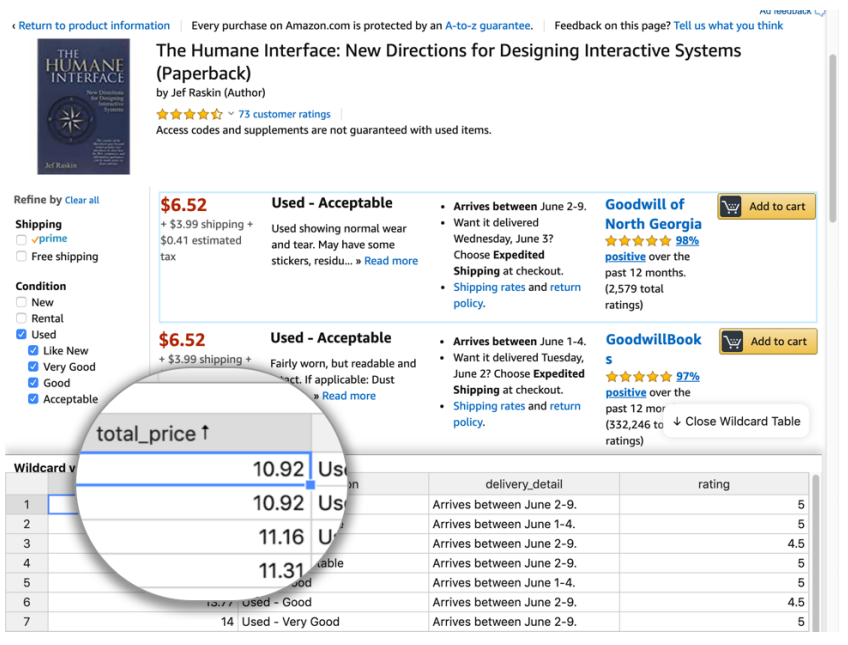

Figure 5. Sorting the used sellers page on Amazon by total price, including fees. The original page doesn't have sorting, and doesn't show the combined price.

\subsection{Range of Customizations}

We have found that data-driven customization can serve a broad range of useful purposes. Here we expand on some archetypal examples that illuminate aspects of using the system in practice.

4.1.1 Sorting and Filtering. It might seem that most websites already have adequate sorting and filtering functionality, but we have found it surprisingly helpful to add new sorting/filtering functionality to websites using Wildcard.

Sometimes, websites have opaque ranking algorithms which presumably maximize profit but restrict user agency. For example, Airbnb previously allowed users to sort listings by price, but removed that feature in 2012. In other cases, a lack of sorting options seems more like an innocent omission; for example, the Instacart grocery delivery service has a spartan UI for viewing an order, which doesn't allow for sorting items by price or category. In both of these cases, Wildcard enables users to take back some control.

In the current implementation of Wildcard, users can only sort and filter entries that are shown on the current page, which means that users are not entirely liberated from the site's original ranking. This restriction could be overcome in the future by scraping content across multiple pages, or by using an integrated adapter and avoiding scraping altogether. However, we've also realized that sorting/filtering a single page of a paginated list is sometimes an acceptable outcome (and even a preferable one). It's more useful, for example, to sort 30 recommended Youtube videos than to try to sort all videos on Youtube.

4.1.2 Annotating. Many web annotation systems focus on annotating text or arbitrary webpage content, but Wildcard limits annotations to structured objects extracted by an 
Table 1. A list of data-driven customizations that we have implemented using Wildcard.

\begin{tabular}{llrl}
\hline Website & Description & LOC & Example customizations \\
\hline Airbnb & Travel & 73 & Add Walkability Scores to listings. Sort listings by price. \\
Amazon & Online shopping & 99 & Sort third party sellers by total price, including fees. \\
Blogger & Blogging & 36 & Use alternate text editor to edit blog posts. \\
Expedia & Travel & 41 & Use alternate datepicker to enter travel dates. \\
Flux & Data portal & 67 & Use Wildcard as a faster table editor for editing lab results. \\
Github & Code repository & 62 & Sort a user's code repositories by stars to find popular work. \\
Hacker News & News & 69 & Add read times to links. Filter out links that have been read. \\
Instacart & Grocery delivery & 48 & Sort groceries by price and category. Take notes on items. \\
Uber Eats & Food delivery & 117 & Sort/filter restaurants by estimated delivery ETA and price. \\
Weather.com & Weather & 51 & Sort/filter hourly weather to find nice times of day. \\
Youtube & Videos & 80 & Sort/filter videos by length, to find short videos to watch. \\
\hline
\end{tabular}

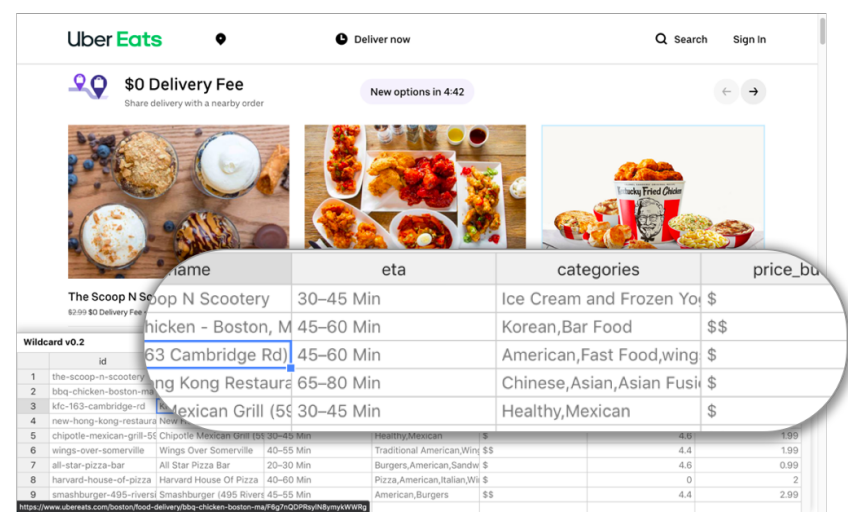

Figure 6. Organizing takeout restaurants on Uber Eats by delivery ETA and price

adapter, resulting in a different set of use cases. Annotating with Wildcard has proven most useful when taking notes on a list of possible options (e.g., evaluating possible Airbnb locations to rent). We have also used it with Instacart's online grocery cart, for jotting down notes as we review an order and consider modifications (shown in Figure 7).

4.1.3 Formulas. Formulas are the most powerful part of the Wildcard system. So far, our language supports only a small number of predefined functions. Adding more should allow a broad range of useful computations, as shown by the success of spreadsheets.

Formulas are especially useful for fetching data from Web APIs. We've used them to augment Airbnb listings with walkability scores, and to augment Hacker News articles with estimated read times as shown in Section 2. One challenge of the current language design is that supporting a new web API requires writing Javascript code to add a new function to the language, because web APIs typically return complex JSON data structures that can't be easily displayed in a single table cell. In the future we would like to make it possible to call new APIs without adding a dedicated function, which

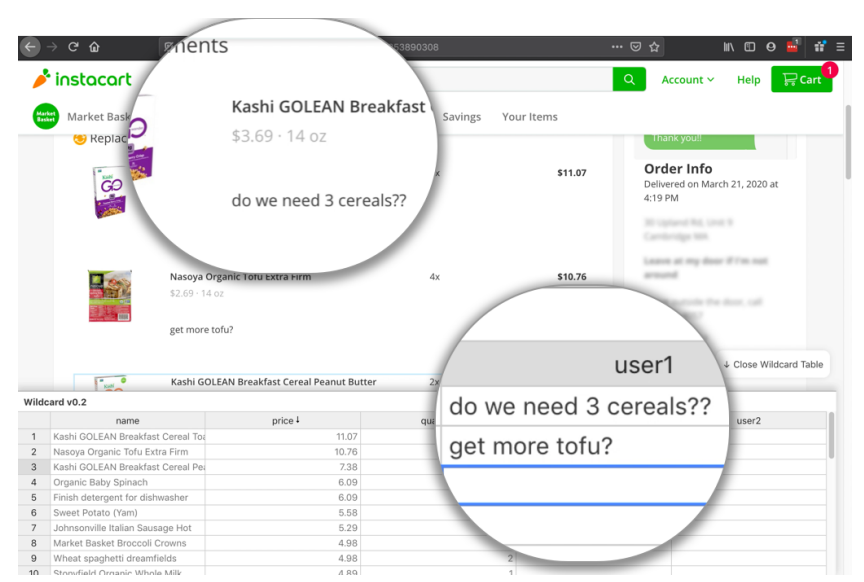

Figure 7. Taking notes on Instacart grocery items, after sorting them by price

might require adding functions to the formula language that can manipulate JSON data.

We have also found instances where simple data manipulation is useful, e.g. transforming the results of an API call with basic arithmetic and string operations, as shown in Section 2.

4.1.4 Cell Editors. We developed two cell editors: custom UI widgets for editing values in the table.

One benefit that cell editors provide is enabling users to incorporate their private information into a web UI. We created a datepicker widget (based on the FullCalendar plugin), which can load calendar data from a Google Calendar. This makes it convenient to enter dates into a website based on the user's personal calendar information, without needing to upload a user's calendar to the website itself.

Another benefit is that a user can choose a single preferred widget for editing a certain type of information across different sites. For example, a user could use their favorite rich text editor to edit text in various websites like blogging platforms and task trackers. To demonstrate this capability, we 


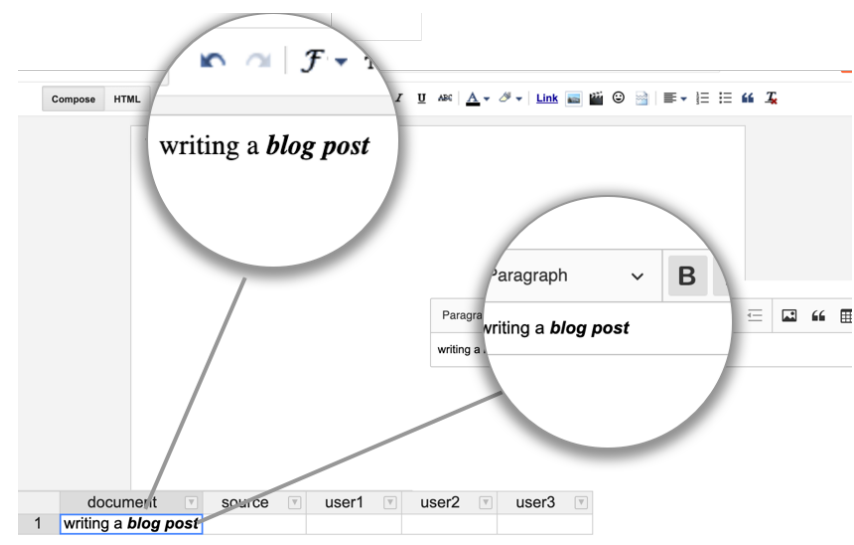

Figure 8. Using a custom text editor widget to edit a blog post on Blogger. The text is synchronized with the Blogger editor through a table cell.

built a text editor based on the CKEditor rich text editor. We used the editor with Google's Blogger website, by building a site adapter that represented the contents of a blog post as a single table cell containing an HTML string (shown in Figure 8).

4.1.5 Limitations. There are many customizations that are not possible to implement with data-driven customization. Some of the limitations are specific to the current implementation of the Wildcard extension, but others are more fundamental to the general paradigm.

One limitation is that Wildcard can only make customizations that use the available data exposed in the table. If the adapter doesn't expose some piece of data, the user can't use it in their customization. The table data format also rules out customizing certain sites that don't have a way to map to a table. The UI modifications available in Wildcard are also limited in scope; deleting arbitrary buttons isn't possible, for example. There is no facility for running automations when the user isn't actively viewing a page-at one point, we wanted to build an automation to repeatedly load a grocery delivery website to check for open delivery slots, but it didn't seem possible to achieve this in Wildcard. We consider these limitations acceptable, since our goal is to support as many useful customizations as possible with a low threshold of difficulty, and not to span all possible customizations.

We have found that one benefit of showing structured data is predictability: once we build an adapter for a website, it is clear what data is available or unavailable for use in customizations. Also, there is sometimes a way to reframe an imperative script in terms of our direct manipulation model. For example, a script that iterates through rows in a page adding some additional information to each row can be reproduced using a single formula in Wildcard.

\subsection{Viability of Scraping}

Separately from the range of customizations, we also evaluated the feasibility of building DOM scraping adapters in practice. In order for third-party customization through Wildcard to succeed, it is important that creating adapters for existing websites takes minimal effort.

Nearly all of our DOM scraping adapters were created by members of our team. However, an external developer unaffiliated with the project contributed one adapter, designed to sort the Github page listing a user's repositories, and they described the experience as "very straightforward."

The adapters for our test sites ranged from 36 to 117 lines of code, averaging 68 lines; Table 1 shows the number of lines of code for each adapter. Most of the code in the adapters is simply using DOM APIs and CSS selectors to implement conventional web scraping logic.

Some of the challenges of writing a DOM scraping adapter are the same ones as with writing normal web scraping code. Sometimes, addressing the desired set of elements can be difficult, and when sites change, scrapers can break; we observed several instances where sites changed their CSS classes and caused Wildcard adapters to no longer work. One benefit of a library of shared wrappers is that if many customizations depend on some piece of scraping logic, rather than having the scraping logic embedded in a single browser extension, it should be more likely to be fixed quickly.

The interactive nature of Wildcard also introduces additional challenges beyond normal web scraping. One challenge is registering appropriate event handlers to update the table data in response to UI changes that happen after initial page load. Another challenge is persisting updates to the DOM-some websites use virtual DOM frameworks that can occasionally overwrite changes made by Wildcard. So far, in practice we've managed to work around these issues for all of the websites we've tried, but we don't claim that any website can be customized through DOM scraping. As web frontend code gets increasingly complex (and starts to move beyond the DOM to other technologies like Shadow DOM or even WebGL), it may become increasingly difficult to customize websites from the outside without first-party support.

AJAX scraping proved very useful in several cases. The Uber Eats website was challenging to scrape because it has a complex DOM structure with machine-generated CSS classes, but the site also uses AJAX requests which contain all the relevant data in a structured form that is much easier to extract. We also found examples where relevant information wasn't present in the DOM at all. On the grocery delivery site Instacart, we found that AJAX requests contained useful information not shown in the UI, like the category and barcode ID of an item. 


\section{Vision}

We envision data-driven customization as a broad paradigm that could extend well beyond the Wildcard proof-of-concept, and ultimately result in new software architectures that empower end users to mold software to their specific needs. Here we explore some of the deeper ideas underlying our work, and future possibilities beyond the Wildcard tool.

\subsection{Decoupling Data from Applications}

When data is freely available outside the context of a specific application, users have more freedom to choose a suitable application for their needs. For example, an RSS feed can be consumed by many reader applications-a journalist can use a power tool optimized for skimming hundreds of news sources a day, while a casual reader can use a simple app to keep up with a few blogs. Motivated users can even create their own custom workflows for filtering and combining RSS feeds, either via traditional programming or in an end-user programming environment like Yahoo Pipes.

However, on the Web today, data is often siloed and only accessible through a single prescribed application. A Facebook feed can only be viewed through the Facebook application. Podcasts, originally served openly through RSS, are beginning to become exclusive to specific platforms like Spotify. This coupling between data and applications leaves users at the mercy of using a single client optimized for specific purposes (e.g., maximizing engagement) that may not be aligned with users' individual desires.

Some services provide APIs that mitigate these siloing effects, but APIs fail to provide a full solution to the problem. First, APIs often provide limited access, especially when an open client ecosystem would harm the economic incentives of a web service built on advertising in a first-party clientin 2012, Twitter infamously imposed restrictions on thirdparty applications that mimicked the "mainstream Twitter consumer client experience." A second problem is that web APIs have a high barrier to entry-they tend to be designed more for programmers creating entire applications or heavyduty automations than for end users casually modifying their own experience.

There have been compelling suggestions for more decentralized architectures that would give users more control of their data. Local-first software [15] suggests that productivity applications should run logic and store data locally, while retaining the benefits of realtime collaboration through peerto-peer synchronization. The SOLID project [6] envisions a decentralized future where users store data on their own servers, and choose to grant limited access to applications. We see data-driven customization as aligned with these decentralized visions, but complementary to them in two ways.

Incrementality: Rather than proposing that web services be totally rearchitected, data-driven customization suggests a more incremental path for adding user agency to existing software.

Data-driven customization allows for lightly augmenting a centralized website with decentralized data storage. Section 2 demonstrated how a user's private annotations on a news site could be stored in their browser, without needing to upload the annotation to the website's server. A hybrid storage model is reasonable here: a centralized storage model makes sense for most of the information on Hacker News that is viewed by all users, but a user's private annotations can easily just be stored in their browser. This model could even be extended with peer-to-peer sharing-a column in a Wildcard table could be shared directly among friends, providing shared annotation of a common website without needing to use the website itself as a centralized intermediary.

By using third-party data extraction, data-driven customization also works with existing websites that do not expose structured data to the user. Ultimately, in adversarial situations where websites are strongly incentivized to restrict access to their data, scraping is unlikely to be a sustainable solution. But we hypothesize that there are many more situations where websites are neutral: not opposed to the idea of end user customization, but also not sufficiently motivated to create and maintain a public API. We see these neutral situations as a context where this kind of incremental approach could succeed. Perhaps some of these websites might be more motivated to provide official extension hooks if they saw the value that users were getting from unofficial community-provided ones.

Focus on end user customization: Having access to the data is a necessary but not sufficient condition for empowering end users to craft their own software experience. Another key ingredient is providing usable tools and interfaces for working with the data.

In data-driven customization, we focus heavily on this part of the solution. By showing raw data in the context of a user interface and allowing small tweaks to the original application's behavior, we provide a smooth path for people to move from using an application to tweaking it.

In this sense, data-driven customization is a complementary approach to other projects that focus on getting users greater access to their data. In a decentralized future where data is stored locally rather than in cloud silos, interfaces like Wildcard would be one technique for actually making use of this data in service of greater end user flexibility.

\subsection{Customization by Direct Manipulation}

Hutchins, Hollan and Norman [13] define a direct manipulation interface as one that uses a model-world metaphor rather than a conversation metaphor. Instead of presenting an "assumed" but not directly visible world that the user converses with, "the world is explicitly represented" and the user can "[act] upon the objects of the task domain themselves." 
Although most GUIs today employ direct manipulation, software customization tools typically use an imperative programming model, which implements the conversational metaphor rather than direct manipulation. Here, for example, is how a user retrieves a list of of calendar names from the Calendar application in Applescript [10], the scripting language for customizing Mac OS applications:

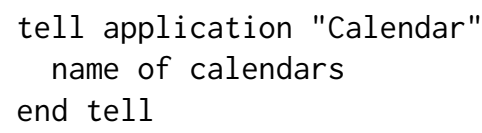

Some customization environments like Mac Automator and Zapier forego textual syntax and let the user connect programs and construct automations by dragging and dropping icons representing commands. These environments still do not constitute direct manipulation, though: the objects being manipulated are in the domain of programming, not in the domain of the task at hand.

Imperative programming is a reasonable choice as the model for building customizations. Turing-complete programming provides a high ceiling for possible customizations, and a sequence of commands is a natural fit for automations that simulate a series of steps taken by the user. There is, however, a serious drawback to this approach. MacLean et al. [19] describe an ideal for user-tailorable systems: a "gentle slope" from using to customizing, where small incremental increases in skill lead to corresponding increments of customization power. Requiring users wanting to customize their applications to learn programming creates an abrupt "cliff," exacting a significant investment in learning even to implement the simplest customizations. Another goal of MacLean et al. is to make it "as easy to change the environment as it is to use it"-at least for some subset of changes. But in scripting languages, the experience of customization does not remotely resemble the experience of use.

With data-driven customization we aim to provide a gentler slope, by using direct manipulation for software customization. The data shown in the table view is the domain data from the original application. The user makes changes to the data by selecting areas of interest in the table, e.g. sorting/filtering by clicking the relevant column header, or adding annotations by clicking and typing on the relevant row. At every step, the user receives intermediate feedback, not only in the table view, but also in the original application, so it's clear whether they are making progress towards their desired result. These types of interactions are common in GUI applications, and Wildcard therefore seems to meet MacLean et al.'s goal: some one-click customizations are as easy as using the original application. Formulas introduce some additional complexity, but spreadsheets have demonstrated that formula programming is still accessible to many users, helped by the pure functional semantics and the visibility of intermediate results.
One aspect of directness that we have chosen not to pursue in Wildcard is enabling customization in closer proximity to the original user interface elements, as explored by other tools like Scotty [11]. While closer proximity might be helpful, we have found that augmenting the original UI with a distinct, additional representation provides a more consistent experience across all applications, and clearly shows what structured data is available to work with. We also emphasize the mapping between the representations by highlighting content in the original page, similiar to the way that browser developer tools highlight the currently selected element in the DOM inspector in the original page.

Ainsworth et al. provide a helpful taxonomy of the value of multiple representations [1]. In their terms, Wildcard plays a complementary role by supporting a different set of tasks from the original application, while displaying shared information. Wildcard may also help construct deeper understanding by subtraction: by stripping away details and only showing the essential data in an interface, Wildcard encourages thinking of an application in terms of its core information, rather than the specific capabilities provided by the current user interface. In our experience, we've often found that looking at a site's data in table format tends to spur new ideas for customizations which weren't evident from looking at the original UI.

\subsection{Semantic Wrappers}

Ad hoc customization tools enable customization without using official extension APIs, enabling a broader range of customizations on top of more applications. For example, web browser extensions have demonstrated the utility of customizing websites through manipulating the DOM, without websites needing to provide explicit extension APIs. However, ad hoc customization comes with a cost: these tools typically operate at a low level of abstraction, e.g. manipulating user interface elements, rather than in a meaningful domain model. This makes it harder for end users to write scripts, and makes the resulting scripts more brittle as the specifics of a user interface change.

Anticipated customization tools, in contrast, use explicit extension APIs provided by the application developer. Examples of this include accessing a backend web API, or writing a customization in Applescript for an application that exposes its domain model to the scripting language. The main benefit of this style is that it allows the extension author to work with meaningful concepts in the application domain-"create a new calendar event" rather than "click the button that contains the text "new event" "-which makes customizations easier to build and more robust. However, the plugin API limits the types of customizations that can be built, and many applications don't have any plugin API.

With Wildcard, we use a hybrid approach that aims to provide the best of both worlds. Third-party programmers 
implement site-specific adapters that are internally implemented as ad hoc customizations, but externally provide a high-level interface to the application, abstracting away the details of the user interface. These wrappers are added to a shared repository, available to all users of the system. When an end user is using a site that already has an adapter, they benefit from a semantic customization experience that avoids low-level details.

One way to view this approach is as introducing a new abstraction barrier into third-party extension. Typically, a third-party customization script combines two responsibilities: 1) mapping the low-level details of a user interface to semantic constructs (e.g., using CSS selectors to find certain page elements), and 2) handling the actual logic of the specific customization. Even though the mapping logic is often more generic than the specific customization, the intertwining of these two responsibilities in a single script makes it very difficult to share the mapping logic across scripts.

With Wildcard we propose a decoupling of these two layers: a repository of shared wrappers maintained by programmers, and a separate repository of specific customizations built on top of these wrappers. This general architecture has been successfully demonstrated by projects like Gmail.js, an open source project that wraps the Gmail web client in a convenient API for browser extensions to build on.

The success of semantic wrappers depends on a key hypothesis: that a single wrapper created by a programmer can be used for many different purposes by end users. Although we've validated that a single generic adapter can support many customizations, so far the people making the adapters have largely been the same people building customizations on top of them, so more work is needed to fully test this hypothesis.

The distribution mechanism for semantic wrappers is also important for encouraging an ecosystem of shared wrappers. Currently, the distribution mechanism is simply merging the code for all adapters into the main Wildcard codebase. This is a simple solution, but makes it fairly difficult to contribute new wrappers and requires installing a new version of the extension to gain access to new warppers. In the future we might explore other mechanisms, like an online repository that the extension pulls from dynamically. Security is also a consideration-DOM scraping adapters can execute arbitrary Javascript code, which means a malicious adapter could exfiltrate sensitive information from a page. Approaches to security could include centralized code review, using a restricted scraping DSL, or creating a sandboxed context for scrapers without access to networking APIs.

\subsubsection{Alternate Mechanisms for Wrapper Creation. Re-} quiring programming to create wrappers has an obvious limitation. If an end user wants to customize a site and no programmer has contributed a wrapper for that site, then they have no means of customizing it. Although a sufficiently vibrant community of programmers could produce wrappers for many popular sites, it's unrealistic to imagine covering all websites that end users might want to customize. We envision two strategies for dealing with this problem:

End user wrapper creation: If end users could create wrappers without programming, they could customize any website, as long as it worked with the wrapper creation process. We could try integrating techniques from projects like Helena [9] which enable end users to scrape websites by demonstration. Another intriguing possibility we plan to explore in the future is blurring the boundaries between scraping and customizing by using spreadsheet formulas as a means of guiding the scraping process.

First-party wrapper creation: An integrated adapter installed by the developer of an application could directly access internal state, providing the same functionality as a DOM scraping adapter but in a more robust way.

With the advent of rich frontend web frameworks, structured application state is now often available in the web client. We suspect it is possible to create plugins for frontend frameworks that expose this state to Wildcard with only minimal effort from the application developers. This kind of plugin would allow developers to integrate with an ecosystem of formulas and customization tools without needing to build that functionality from scratch.

\section{Related Work}

This paper extends work reported in our workshop paper that presented an early version of the Wildcard extension [18] . We have substantially extended that work by creating the table adapter abstraction, reimplementing the internals of Wildcard around that abstraction, evaluating the system on many more websites and use cases, and by characterizing the design of the system in more detail.

Data-driven customization relates to two broad areas of related work. Our problem statement is related to software customization tools, and our solution approach is related to spreadsheets and other direct manipulation interfaces.

\subsection{Customization Tools}

Data-driven customization is most closely related to other tools that aim to empower end users to customize software without traditional coding.

This lineage goes back at least to the Buttons system by MacLean et al. [19], where Xerox Lisp users could share buttons that performed various "tailoring" actions on the system. The authors proposed the "gentle slope" idea which has greatly influenced our approach to data-driven customization (as discussed in Section 5.2). The authors also point out the importance of a "tailoring culture" where people with different skillsets collaborate to produce useful customizations; in their system, Lisp programmers create buttons that 
others can use, modify, and rearrange. This division of labor corresponds to our idea of semantic wrappers, where end user customization is supported by programmer-created building blocks.

More recently, Tchernavskij introduced the notion of malleable software, which aims to allow users to tailor their software by pulling apart and recombining individual user interface elements [22]. This work builds on prior work on customizing graphical interfaces, including Beaudouin-Lafon's idea of instrumental interaction [4] and Klokmose et al.'s customizable Webstrates environment [16]. Data-driven customization shares the overall goals of this line of work, and proposes new interaction techniques for achieving them. In particular, by showing users a structured data representation and allowing them to perform lightweight programming through formulas, our approach supports customizations of intermediate complexity: more sophisticated than those that can be achieved merely by manipulating existing interface elements, but simple enough to not require full-blown programming.

Other web customization tools have also aimed to enable end users to modify web interfaces without programming. Sifter [14] enables end users to sort and filter lists of data obtained by web scraping, much like Wildcard's sorting features. The main difference between the systems is that data-driven customization has many other use cases besides sorting and filtering. Also, Sifter involves end users in a semi-automated data extraction process, rather than having programmers create wrappers. This provides coverage of more websites, but at the expense of complicating the end user experience. We might integrate end user scraping techniques in Wildcard in the future, but we believe that, when possible, it is valuable for end users to have a customization experience decoupled from the challenge of scraping the underlying data. Sifter also implements scraping across multiple pages, a valuable feature for sorting and filtering that isn't present in Wildcard.

Thresher [12] helps end users create wrappers that map website content to Semantic Web schemas like "Movie" or "Director," and augments websites with new functionality by exploiting that schema information. Wildcard shares the general idea of wrappers, but maps to a generic table data type rather than more specific schemas, increasing the range of supported data and allowing for a simpler mapping process.

There are many software customization tools that offer simplified forms of programming for end users. Chickenfoot [7] and Coscripter [17] offer user friendly syntax for writing web automation scripts; Applescript [10] has a similar goal for desktop customization. There are visual programming environments for customization that don't involve writing any text: Automator for Mac and Shortcuts for iOS are modern options for customizing Apple products, and Zapier enables users to connect different web applications together visually.
As mentioned previously, these tools all require writing imperative programs, in contrast to the more declarative and direct approach of data-driven customization.

\subsection{Spreadsheets and Visual Query Interfaces}

Another relevant area involves spreadsheets and visual query interfaces. We take inspiration from these tools in our work, but apply them in a different domain: customizing existing software applications, rather than interacting with databases or constructing software from scratch.

The most closely related work is in systems that offer spreadsheet-like querying of relational data. SIEUFERD by Bakke and Karger [3] is one such recent system, and their paper presents a survey of many other similar tools. Our work is particularly influenced by the authors' observation that a user should be able to modify queries by interacting with the results of the query rather than some representation of the query itself. SIEUFERD's interface supports a far more general range of queries than Wildcard, but the core principles of the user interface are the same. Airtable is another example of a modern commercial product that offers spreadsheet-like interaction with a relational database.

Our work is also inspired by the many projects that have explored using spreadsheets as a foundation for building software applications, including Object Spreadsheets [20], Quilt [5], Gneiss [8], Marmite [23], and Glide. We share the main idea of connecting a spreadsheet view to a GUI, but we apply it to software customization, rather than building software from scratch.

Another related system is ScrAPIr, by Alrashed et al. [2], which enables end users to access backend web APIs without programming. ScrAPIr shares our high level goal of end user empowerment, as well as the idea of wrappers, by creating a shared library of wrappers around existing web APIs. Unlike Wildcard, however, ScrAPIr targets explicit APIs exposed by developers. It also focuses on backend services and doesn't aim to extend the frontend interfaces of web applications.

\section{Conclusion and Future Work}

A primary goal for future work is to evaluate the system with more users. What usability barriers do people face? What types of customizations do they choose to create? What formulas prove most helpful? We hope to answer these questions through real deployment of the tool.

Another future goal is to better characterize the limits of the table-editing paradigm. Are there ways to offer an increase in power and functional complexity, while retaining a programming model that is simpler for end users than conventional coding? For example, we could enable users to set up triggers to perform actions like sending notifications when certain conditions are met in the table view.

Finally, more broadly, data-driven customization suggests new possibilities for how multiple applications might be 
integrated in new ways, by synchronizing their underlying data representations in a shared format. So far, this work has mostly explored the implications for customization within a single application, but it would be interesting to explore how end users could use these techniques to synchronize data across applications to avoid manual coordination work.

As computing plays an ever greater role in people's lives, it is increasingly important that users have agency over the behavior of their software, rather than having every detail be dictated by companies whose interests are not always aligned with their own. Data-driven customization, we hope, is a step on the path from normal use to deep modification, in support of a more adaptable experience for all users.

\section{Acknowledgments}

Thank you to Tarfah Alrashed, Glen Chiacchieri, David Karger, Steve Krouse, Rob Miller, Santiago Perez De Rosso, Arvind Satyanarayan, Daniel Windham, and Maggie Yellen, who all provided helpful feedback on this work. We also thank our anonymous reviewers, whose insightful comments greatly improved this paper.

This research was funded in part by the International Design Center, a collaboration between MIT and SUTD (the Singapore University of Technology and Design), and by the Secure and Trustworthy Cyberspace (SaTC) program of the CISE division of the National Science Foundation under Award Number 1801399.

\section{References}

[1] Shaaron Ainsworth. 1999. The Functions of Multiple Representations. Computers \& Education 33, 2-3 (Sept. 1999), 131-152. https://doi.org/ 10.1016/S0360-1315(99)00029-9

[2] Tarfah Alrashed, Jumana Almahmoud, Amy X. Zhang, and David R. Karger. 2020. ScrAPIr: Making Web Data APIs Accessible to End Users. In Proceedings of the 2020 CHI Conference on Human Factors in Computing Systems (CHI '20). Association for Computing Machinery, Honolulu, HI, USA, 1-12. https://doi.org/10.1145/3313831.3376691

[3] Eirik Bakke and David R. Karger. 2016. Expressive Query Construction through Direct Manipulation of Nested Relational Results. In Proceedings of the 2016 International Conference on Management of Data SIGMOD '16. ACM Press, San Francisco, California, USA, 1377-1392. https://doi.org/10.1145/2882903.2915210

[4] Michel Beaudouin-Lafon. 2000. Instrumental Interaction: An Interaction Model for Designing Post-WIMP User Interfaces. In Proceedings of the SIGCHI Conference on Human Factors in Computing Systems (CHI '00). ACM, New York, NY, USA, 446-453. https: //doi.org/10.1145/332040.332473

[5] Edward Benson, Amy X. Zhang, and David R. Karger. 2014. Spreadsheet Driven Web Applications. In Proceedings of the 27th Annual ACM Symposium on User Interface Software and Technology - UIST '14. ACM Press, Honolulu, Hawaii, USA, 97-106. https://doi.org/10.1145/ 2642918.2647387

[6] Tim Berners-Lee. 2019. One Small Step for the Web.... https://medium.com/@timberners_lee/one-small-step-for-theweb-87f92217d085.

[7] Michael Bolin, Matthew Webber, Philip Rha, Tom Wilson, and Robert C. Miller. 2005. Automation and Customization of Rendered Web Pages. In Proceedings of the 18th Annual ACM Symposium on User Interface
Software and Technology - UIST '05. ACM Press, Seattle, WA, USA, 163. https://doi.org/10.1145/1095034.1095062

[8] Kerry Shih-Ping Chang and Brad A. Myers. 2014. Creating Interactive Web Data Applications with Spreadsheets. In Proceedings of the 27th Annual ACM Symposium on User Interface Software and Technology UIST '14. ACM Press, Honolulu, Hawaii, USA, 87-96. https://doi.org/ 10.1145/2642918.2647371

[9] Sarah E. Chasins, Maria Mueller, and Rastislav Bodik. 2018. Rousillon: Scraping Distributed Hierarchical Web Data. In The 31st Annual ACM Symposium on User Interface Software and Technology - UIST '18. ACM Press, Berlin, Germany, 963-975. https://doi.org/10.1145/3242587. 3242661

[10] William R. Cook. 2007. AppleScript. In Proceedings of the Third ACM SIGPLAN Conference on History of Programming Languages - HOPL III. ACM Press, San Diego, California, 1-1-1-21. https://doi.org/10.1145/ 1238844.1238845

[11] James R. Eagan, Michel Beaudouin-Lafon, and Wendy E. Mackay. 2011. Cracking the Cocoa Nut: User Interface Programming at Runtime. In Proceedings of the 24th Annual ACM Symposium on User Interface Software and Technology - UIST '11. ACM Press, Santa Barbara, California, USA, 225. https://doi.org/10.1145/2047196.2047226

[12] Andrew Hogue and David Karger. 2005. Thresher: Automating the Unwrapping of Semantic Content from the World Wide Web. In Proceedings of the 14th International Conference on World Wide Web-WWW'05. ACM Press, Chiba, Japan, 86. https://doi.org/10.1145/1060745.1060762

[13] Edwin L Hutchins, James D Hollan, and Donald A Norman. 1985. Direct Manipulation Interfaces. (1985), 28.

[14] David F. Huynh, Robert C. Miller, and David R. Karger. 2006. Enabling Web Browsers to Augment Web Sites' Filtering and Sorting Functionalities. In Proceedings of the 19th Annual ACM Symposium on User Interface Software and Technology - UIST '06. ACM Press, Montreux, Switzerland, 125. https://doi.org/10.1145/1166253.1166274

[15] Martin Kleppmann, Adam Wiggins, Peter van Hardenberg, and Mark McGranaghan. 2019. Local-First Software: You Own Your Data, in Spite of the Cloud. In Proceedings of the 2019 ACM SIGPLAN International Symposium on New Ideas, New Paradigms, and Reflections on Programming and Software - Onward! 2019. ACM Press, Athens, Greece, 154-178. https://doi.org/10.1145/3359591.3359737

[16] Clemens N. Klokmose, James R. Eagan, Siemen Baader, Wendy Mackay, and Michel Beaudouin-Lafon. 2015. Webstrates: Shareable Dynamic Media. In Proceedings of the 28th Annual ACM Symposium on User Interface Software \& Technology - UIST '15. ACM Press, Daegu, Kyungpook, Republic of Korea, 280-290. https://doi.org/10.1145/2807442.2807446

[17] Gilly Leshed, Eben M. Haber, Tara Matthews, and Tessa Lau. 2008. CoScripter: Automating \& Sharing How-to Knowledge in the Enterprise. In Proceedings of the SIGCHI Conference on Human Factors in Computing Systems (CHI '08). ACM, New York, NY, USA, 1719-1728. https://doi.org/10.1145/1357054.1357323

[18] Geoffrey Litt and Daniel Jackson. 2020. Wildcard: Spreadsheet-Driven Customization of Web Applications. In Companion Proceedings of the 4th International Conference on the Art, Science, and Engineering of Programming. Association for Computing Machinery, Porto, Portugal., 10. https://doi.org/10.1145/3397537.3397541

[19] Allan MacLean, Kathleen Carter, Lennart Lövstrand, and Thomas Moran. 1990. User-Tailorable Systems: Pressing the Issues with Buttons. In Proceedings of the SIGCHI Conference on Human Factors in Computing Systems Empowering People - CHI '90. ACM Press, Seattle, Washington, United States, 175-182. https://doi.org/10.1145/97243.97271

[20] Matt McCutchen, Shachar Itzhaky, and Daniel Jackson. 2016. Object Spreadsheets: A New Computational Model for End-User Development of Data-Centric Web Applications. In Proceedings of the 2016 ACM International Symposium on New Ideas, New Paradigms, and Reflections on Programming and Software - Onward! 2016. ACM Press, Amsterdam, Netherlands, 112-127. https://doi.org/10.1145/2986012.2986018 
[21] B. Shneiderman. 1983. Direct Manipulation: A Step Beyond Programming Languages. Computer 16, 8 (Aug. 1983), 57-69. https: //doi.org/10.1109/MC.1983.1654471

[22] Philip Tchernavskij. 2019. Designing and Programming Malleable Software. PhD Thesis. Université Paris-Saclay, École doctorale $\mathrm{n}^{\circ} 580 \mathrm{Sci}$ ences et Technologies de l'Information et de la Communication (STIC).
[23] Jeffrey Wong and Jason I. Hong. 2007. Making Mashups with Marmite: Towards End-User Programming for the Web. In Proceedings of the SIGCHI Conference on Human Factors in Computing Systems - CHI '07. ACM Press, San Jose, California, USA, 1435-1444. https://doi.org/10. $1145 / 1240624.1240842$ 